Draft version August 29, 2018

Preprint typeset using $\mathrm{IAT}_{\mathrm{E}} \mathrm{X}$ style emulateapj v. 5/2/11

\title{
INTERMITTENCY AND ALIGNMENT IN STRONG RMHD TURBULENCE
}

\author{
B. D. G. Chandran ${ }^{1,2}$, A. A. Schekochinin ${ }^{2,3}$, and A. Mallet $^{3}$ \\ Draft version August 29, 2018
}

\begin{abstract}
We develop an analytic model of intermittent, three-dimensional, strong, reduced magnetohydrodynamic (RMHD) turbulence with zero cross helicity. We take the fluctuation amplitudes to have a $\log$-Poisson distribution and incorporate into the model a new phenomenology of scale-dependent dynamic alignment between the Elsässer variables $\boldsymbol{z}^{ \pm}$. We find that the structure function $\left\langle\left|\Delta \boldsymbol{z}_{\lambda}^{ \pm}\right|^{n}\right\rangle$ scales as $\lambda^{1-\beta^{n}}$, where $\Delta \boldsymbol{z}_{\lambda}^{ \pm}$is the variation in $\boldsymbol{z}^{ \pm}$across a distance $\lambda$ perpendicular to the magnetic field. We calculate the value of $\beta$ to be $\simeq 0.69$ based on our assumptions that the energy cascade rate is independent of $\lambda$ within the inertial range, that the most intense coherent structures are two-dimensional with a volume filling factor $\propto \lambda$, and that most of the cascade power arises from interactions between exceptionally intense fluctuations and much weaker fluctuations. Two consequences of this structure-function scaling are that the total-energy power spectrum is $\propto k_{\perp}^{-1.52}$ and that the kurtosis of the fluctuations is $\propto \lambda^{-0.27}$. Our model resolves the problem that alignment angles defined in different ways exhibit different scalings. Specifically, we find that the energy-weighted average angle between the velocity and magnetic-field fluctuations is $\propto \lambda^{0.21}$, the energy-weighted average angle between $\Delta \boldsymbol{z}^{+}$and $\Delta \boldsymbol{z}^{-}$is $\propto \lambda^{0.10}$, and the average angle between $\Delta \boldsymbol{z}^{+}$and $\Delta \boldsymbol{z}^{-}$without energy weighting is $\propto[\ln (L / \lambda)]^{-1 / 2}$ when $L / \lambda \gg 1$, where $L$ is the outer scale. These scalings appear to be consistent with numerous results from direct numerical simulations.

Subject headings: magnetohydrodynamics — turbulence — plasmas — solar wind
\end{abstract}

\section{INTRODUCTION}

Plasma turbulence plays an important role in many astrophysical systems, including accretion flows around black holes, intracluster plasmas in clusters of galaxies, and outflows from stars, including the solar wind. In many of these systems, the energetically dominant component of the turbulence is non-compressive and can be modeled, at least in an approximate way, within the framework of incompressible magnetohydrodynamics (MHD).

In incompressible MHD, velocity and magnetic-field fluctuations $(\delta \boldsymbol{v}$ and $\delta \boldsymbol{B})$ propagate either parallel or anti-parallel to the local background magnetic field $\boldsymbol{B}_{\mathrm{loc}}$, and nonlinear interactions occur only between counter-propagating fluctuations (Iroshnikov 1963; Kraichnan 1965). As a consequence, the energy cascade is anisotropic, producing small-scale structures or "eddies" that satisfy $\lambda \ll l$, where $l(\lambda)$ is the correlation length of an eddy parallel (perpendicular) to $\boldsymbol{B}_{\text {loc }}$ (Shebalin et al. 1983; Goldreich \& Sridhar 1995; Ng \& Bhattachariee 1996; Galtier et al. 2000; Cho \& Vishniac 2000; Maron \& Goldreich 2001). When $\lambda \ll l$, the components of $\delta \boldsymbol{v}$ and $\delta \boldsymbol{B}$ perpendicular to $\boldsymbol{B}_{\text {loc }}$ evolve independently of the components parallel to $\boldsymbol{B}_{\mathrm{loc}}$ and are well described by reduced MHD (RMHD) (Kadomtsev \& Pogutse 1974; Strauss 1976). When $\delta B \ll B_{\text {loc }}$ and $\rho_{\mathrm{p}} \ll \lambda \ll l$, where $\rho_{\mathrm{p}}$ is the proton gyroradius, RMHD is a rigorous limit of gyroki-

\footnotetext{
${ }^{1}$ Space Science Center and Department of Physics, University of New Hampshire, Durham, NH 03824; benjamin.chandran@unh.edu

${ }^{2}$ Merton College, University of Oxford, Oxford OX1 4JD, United Kingdom

${ }^{3}$ Rudolf Peierls Centre for Theoretical Physics, University of Oxford, Oxford OX1 3NP, United Kingdom
}

netics and is valid for both collisional and collisionless plasmas (Schekochihin et al. 2009).

In this paper, we propose a phenomenological theory of RMHD turbulence that goes beyond scaling theories for spectra (Iroshnikov 1963; Kraichnan 1965; Goldreich \& Sridhar 1995; Boldyrev 2006) and allows us to make predictions concerning the scale dependence of arbitrary-order structure functions and the relative orientation of the turbulent magnetic field and velocity. A new feature of this theory is that it accounts, within one model, for both intermittency and scale-dependent dynamic alignment (SDDA).

The concept of SDDA was introduced by Boldyrev (2005, 2006), who argued that the angle $\phi_{\lambda}$ between $\delta \boldsymbol{v}_{\lambda}$ and $\delta \boldsymbol{B}_{\lambda}$ decreases with decreasing $\lambda$, where $\delta \boldsymbol{v}_{\lambda}$ and $\delta \boldsymbol{B}_{\lambda}$ are the fluctuations in the velocity and magnetic field at perpendicular scale $\lambda$. As $\phi_{\lambda}$ decreases, nonlinear interactions in RMHD weaken, causing the power spectrum of the fluctuation energy to flatten relative to models that neglect SDDA.

Intermittency is the phenomenon in which the fluctuation energy is concentrated into an increasingly small fraction of the volume as $\lambda \rightarrow 0$. Intermittency has been measured in hydrodynamic turbulence (e.g., Benzi et al. 1993), solar-wind turbulence (Burlaga 1991; Horburv \& Balogh 1997; Sorriso-Valvo et al. 1999; Forman \& Burlaga 2003; Bruno et al. 2007; Wan et al. 2012a; Osman et al. 2012; Perri et al. 2012; Osman et al. 2014), numerical simulations of MHD turbulence and RMHD turbulence (Müller \& Biskamp 2000; Maron \& Goldreich 2001; Müller et al. 2003; Beresnvak \& Lazarian 2006; Mininni \& Pouquet 2009; Rodriguez Imazio et al. 2013), and hybrid-Vlasov and particle-in-cell simulations of plasma turbulence (Greco et al. 2012; Servidio et al. 2012; Wan et al. 
2012b; Karimabadi et al. 2013; Wu et al. 2013). A number of theoretical models have been introduced to describe intermittency, including the log-normal model (Kolmogorov 1962; Gurvich \& Yaglom 1967), the "constant- $\beta$ " model (Frisch et al. 1978), and multifractal models in which the fluctuation amplitudes scale differently on different subsets of the volume that have different fractal dimensions (Parisi \& Frisch 1985; Paladin \& Vulpiani 1987). One such multi-fractal model, based on a log-Poisson probability distribution function for the local dissipation rate, was developed by She \& Leveque (1994) (see also Dubrulle 1994). She \& Leveque's (1994) approach served as the basis for several previous studies of intermittency in both compressible and incompressible MHD turbulence (Grauer et al. 1994; Politano \& Pouquet 1995; Müller \& Biskamp 2000; Boldvrev et al. 2002a, b).

We draw upon ideas from the She-Leveque model to construct an analytic model of strong RMHD turbulence that incorporates a new phenomenology of SDDA. We present this model in Section 2. In Section 3, we compare our model with previously published numerical simulations, and in Section 4 we discuss our results and the relation between our work and previous turbulence models.

\section{ANALYTIC MODEL OF STRONG RMHD TURBULENCE}

The equations of incompressible MHD can be written in the form

$$
\frac{\partial \boldsymbol{z}^{ \pm}}{\partial t} \mp \boldsymbol{v}_{\mathrm{A}} \cdot \nabla \boldsymbol{z}^{ \pm}=-\boldsymbol{z}^{\mp} \cdot \nabla \boldsymbol{z}^{ \pm}-\nabla \Pi,
$$

where $\boldsymbol{z}^{ \pm}=\delta \boldsymbol{v} \pm \delta \boldsymbol{B} / \sqrt{4 \pi \rho}$ are the Elsässer variables, $\delta \boldsymbol{v}$ and $\delta \boldsymbol{B}$ are the velocity and magnetic-field fluctuations, $\rho$ is the mass density, $\boldsymbol{v}_{\mathrm{A}}=\boldsymbol{B}_{0} / \sqrt{4 \pi \rho}$ is the Alfvén velocity, $\boldsymbol{B}_{0}$ is the background magnetic field, $\Pi=\left(p+B^{2} / 8 \pi\right) / \rho, p$ is the pressure, $\boldsymbol{B}=\boldsymbol{B}_{0}+\delta \boldsymbol{B}$, and $\nabla \cdot \boldsymbol{z}^{ \pm}=0$. The RMHD equations are equivalent to Equation (1) supplemented by the condition

$$
\boldsymbol{B}_{0} \cdot \boldsymbol{z}^{ \pm}=0 \text {. }
$$

Throughout this paper, we neglect dissipation and focus on the inertial range.

\subsection{Statistical Distribution of Field Increments}

We consider the turbulence to be an ensemble of approximately localized $z^{+}$and $z^{-}$structures. We define

$$
\Delta \boldsymbol{z}_{\lambda}^{ \pm}=\boldsymbol{z}^{ \pm}(\boldsymbol{x}+0.5 \lambda \hat{\boldsymbol{s}}, t)-\boldsymbol{z}^{ \pm}(\boldsymbol{x}-0.5 \lambda \hat{\boldsymbol{s}}, t),
$$

where $\hat{\boldsymbol{s}}$ is a unit vector perpendicular to $\boldsymbol{B}(\boldsymbol{x}, t)$. We define $\delta z_{\lambda}^{ \pm}$to be $\left|\Delta \boldsymbol{z}_{\lambda}^{ \pm}\right|$averaged over the direction of $\hat{\boldsymbol{s}}$, and we define $\theta_{\lambda}$ to be the (positive semi-definite) angle between $\Delta \boldsymbol{z}_{\lambda}^{+}$and $\Delta \boldsymbol{z}_{\lambda}^{-}$averaged over the direction of $\hat{\boldsymbol{s}}$. We think of $\delta z_{\lambda}^{ \pm}(\boldsymbol{x}, t)$ as the characteristic amplitude of the $z^{ \pm}$structure of scale $\lambda$ that is located at position $\boldsymbol{x}$. Nonlinear interactions cause each structure at scale $\lambda$ to break up into a number of structures at smaller scales. These smaller structures in turn break up into even smaller structures, and so on.

As can be seen from Equation (11), $\boldsymbol{z}^{ \pm}$fluctuations propagate with velocity $\mp \boldsymbol{v}_{\mathrm{A}}$. We can thus view $z^{-}$ $\left(z^{+}\right)$structures as wave packets that propagate parallel (anti-parallel) to the background magnetic field while being distorted by nonlinear interactions. The form of the nonlinear term in Equation (1) implies that nonlinear interactions occur only between $z^{+}$fluctuations and $z^{-}$ fluctuations, and not between fluctuations that propagate in the same direction (Iroshnikov 1963; Kraichnan 1965). The energy cascade in RMHD turbulence can thus be viewed as resulting from "collisions" between counterpropagating wave packets. In the discussion below, we use the terms "structure," "wave packet," and "fluctuation" interchangeably.

In the Appendix, we argue that if a $\delta z_{\lambda}^{ \pm}$fluctuation collides with a $\delta z_{\lambda}^{\mp}$ fluctuation that is either much stronger or much weaker than $\delta z_{\lambda}^{ \pm}$, then $\lambda$ changes for both fluctuations (that is, they are sheared by each other), but the fluctuation amplitudes remain approximately the same. We refer to such collisions as "highly imbalanced." On the other hand, if $\delta z_{\lambda}^{+} \sim \delta z_{\lambda}^{-}$("balanced collisions"), then in general both $\lambda$ and the fluctuation amplitudes decrease, as in models of non-intermittent MHD and RMHD turbulence (e.g., Goldreich \& Sridhar 1995).

To construct an analytic model of RMHD turbulence, we assume that each balanced collision reduces a fluctuation's amplitude by a constant factor $\beta$, which satisfies

$$
0<\beta<1
$$

while highly imbalanced collisions reduce $\lambda$ without reducing a fluctuation's amplitude. Thus,

$$
\delta z_{\lambda}^{ \pm}=\overline{\delta z} \beta^{q},
$$

where $\overline{\delta z}$ is the amplitude of the fluctuation's "progenitor" structure at the outer scale (or forcing scale) $L$, and $q$ is the number of balanced collisions experienced by the fluctuation during its evolution from scale $L$ to scale $\lambda$. For simplicity, we set]

$$
\overline{\delta z}=\text { constant. }
$$

To determine a plausible functional form for the probability distribution function (PDF) of $q$, we consider a hypothetical scenario in which balanced collisions have the property that they reduce a fluctuation's amplitude without changing its length scale. In this case, balanced collisions are similar to the "modulation defect events" described by She \& Wavmire (1995), in that a fluctuation's amplitude can be reduced by a finite factor $\beta$ during an interval of time in which $\lambda$ decreases by only an infinitesimal amount. If the length scale of a fluctuation decreases from $L$ to $\lambda$, then we can divide the interval $[0, \ln (L / \lambda)]$ into infinitesimal sub-intervals, and within each sub-interval there is an infinitesimal chance that a modulation defect event occurs. Over the entire interval, however, the average number of modulation defect events is finite. If we assume that the probability of a balanced collision is independent of the number of balanced collisions that have already occurred, then $q$ has a Poisson distribution,

$$
P(q)=\frac{e^{-\mu} \mu^{q}}{q !},
$$

4 More realistically, $\overline{\delta z}$ would have its own (scale-independent) distribution reflecting the non-universal details of the outer-scale statistics (e.g., the statistics of the forcing). 
where $\mu$ is the as-yet-unknown, scale-dependent, mean value of $q$. In RMHD turbulence, balanced collisions do in fact change $\lambda$, and the probability that a balanced collision occurs may depend upon $q$. Thus, the above arguments do not provide a rigorous justification for Equation (77). We proceed, however, using Equation (77) as a model. We further assume that $\mu$ and $\overline{\delta z}$ are the same for $\delta z_{\lambda}^{+}$and $\delta z_{\lambda}^{-}$and thereby restrict our analysis to the case of zero cross helicity.

The median value of $q$ is approximately $\mu$ (Choi 1994), and thus the "typical" value of $\delta z_{\lambda}^{ \pm}$that best characterizes the bulk of the volume is

$$
\delta z_{\lambda}^{*}=\overline{\delta z} \beta^{\mu} .
$$

In contrast, the most intense structures at scale $\lambda$ correspond to $q=0$ and occur with probability $e^{-\mu}$. Equation (51) implies that the variation in $\boldsymbol{z}^{+}$or $\boldsymbol{z}^{-}$ across such a $q=0$ structure is $\overline{\delta z}$, independent of $\lambda$. We assume that these structures correspond to sheetlike quasi-discontinuities (current/vorticity sheets) with a volume-filling factor $\propto \lambda$ (c.f., Grauer et al. 1994; Politano \& Pouquet 1995). Setting $e^{-\mu} \propto \lambda$, we obtain

$$
\mu=A+\ln \left(\frac{L}{\lambda}\right)
$$

where $A$ is a constant that quantifies the breadth of the distribution at the outer scale. We can thus rewrite Equation (8) in the form

$$
\delta z_{\lambda}^{*}=\overline{\delta z}\left(\frac{\lambda}{e^{A} L}\right)^{-\ln \beta} .
$$

\subsection{Timescales and Critical Balance}

We define the nonlinear timescale

$$
\tau_{\mathrm{nl}, \lambda}^{ \pm}=\frac{\lambda}{\delta z_{\lambda}^{\mp} \sin \theta_{\lambda}},
$$

which is the rate at which a $z^{ \pm}$structure at scale $\lambda$ is sheared by the $z^{\mp}$ structure at scale $\lambda$ at that same location. The factor of $\sin \theta_{\lambda}$ is included in Equation (11) because, if $\boldsymbol{z}^{+}$and $\boldsymbol{z}^{-}$are aligned to within a small angle $\theta$, then $\left|\boldsymbol{z}^{\mp} \cdot \nabla \boldsymbol{z}^{ \pm}\right|$is reduced by a factor $\sim \theta$ relative to the case in which $\theta \sim 1$ (Boldvrev 2005).

We define the linear timescale

$$
\tau_{\operatorname{lin}, \lambda}^{ \pm}=\frac{l_{\lambda}^{ \pm}}{v_{\mathrm{A}}}
$$

Here, $l_{\lambda}^{ \pm}$is the "parallel" correlation length of a $\delta z_{\lambda}^{ \pm}$ structure measured along a local mean magnetic field, which is obtained by summing $\boldsymbol{B}_{0}$ with all the magnetic fluctuations at scales that exceed $\lambda$ by a factor of at least a few.

In accord with the critical-balance hypothesis of Goldreich \& Sridhar (1995), we assume that

$$
\tau_{\mathrm{nl}, \lambda}^{ \pm} \sim \tau_{\operatorname{lin}, \lambda}^{ \pm} .
$$

We can rewrite Equation (13) a:

$$
\chi^{ \pm}=\frac{l_{\lambda}^{ \pm} \delta z_{\lambda}^{\mp} \sin \theta_{\lambda}}{\lambda v_{\mathrm{A}}} \sim 1
$$

Equation (13) is also equivalent to the relation

$$
l_{\lambda}^{ \pm} \sim v_{\mathrm{A}} \tau_{\mathrm{nl}, \lambda}^{ \pm},
$$

which states that the parallel correlation length of a $\delta z_{\lambda}^{ \pm}$ fluctuation is roughly the distance it can propagate during its cascade timescale.

When a $\delta z_{\lambda}^{+}$fluctuation collides with a $\delta z_{\lambda}^{-}$fluctuation and $\delta z_{\lambda}^{+} \sim \delta z_{\lambda}^{-} \sim \delta z_{\lambda}^{*}$ (see Equation (8)), nonlinear interactions cause the $\delta z_{\lambda}^{-}$and $\delta z_{\lambda}^{+}$fluctuations to evolve on the same timescale in a strongly coupled and unpredictable way, which impedes the development of alignment. We thus take

$$
\left.\begin{array}{c}
\theta_{\lambda} \sim 1 \\
\tau_{\mathrm{nl}, \lambda}^{ \pm} \sim \lambda / \delta z_{\lambda}^{*}
\end{array}\right\}\left(\text { when } \delta z_{\lambda}^{+} \sim \delta z_{\lambda}^{-} \sim \delta z_{\lambda}^{*}\right) .
$$

The characteristic parallel correlation length of the median-amplitude fluctuations at scale $\lambda$ is then

$$
l_{\lambda}^{*}=\frac{v_{\mathrm{A}} \lambda}{\delta z_{\lambda}^{*}} .
$$

\subsection{Nonlinear Interactions and the Refined Similarity Hypothesis}

Given our assumption that $\tau_{\mathrm{nl}, \lambda}^{ \pm} \sim \tau_{\operatorname{lin}, \lambda}^{ \pm}$, the turbulence is strong, and $\delta z_{\lambda}^{ \pm}$energy cascades to smaller scales on the timescale $\tau_{\mathrm{nl}, \lambda}^{ \pm}$. We define $\epsilon_{\lambda}^{ \pm}$to be the rate at which $z^{ \pm}$energy (per unit mass) is dissipated within a sphere of diameter $\lambda$. In keeping with earlier works on intermittency, we take $\epsilon^{ \pm}$to be "equal in law" to the quantity $\left(\delta z_{\lambda}^{ \pm}\right)^{2} / \tau_{\mathrm{nl}, \lambda}^{ \pm}$(Frisch 1996). This means that the $n^{\text {th }}$ moment of $\epsilon_{\lambda}^{ \pm}$and the $n^{\text {th }}$ moment of the quantity $\left(\delta z_{\lambda}^{ \pm}\right)^{2} / \tau_{\mathrm{nl}, \lambda}^{ \pm}$scale with $\lambda$ in the same way for all $n$. We denote "equality in law" with the symbol $\approx$ and thus write

$$
\epsilon_{\lambda}^{ \pm} \approx \frac{\left(\delta z_{\lambda}^{ \pm}\right)^{2} \delta z_{\lambda}^{\mp} \sin \theta_{\lambda}}{\lambda}
$$

Equation (18) is analogous to Kolmogorov's (1962) refined similarity hypothesis for hydrodynamic turbulence. We note that the scalings that we derive below do not require full "equality in law," but just that the averages of the left- and right-hand sides of Equation (18) scale with $\lambda$ in the same way.

The average dissipation rate within a sphere of diameter $\lambda$ is independent of $\lambda$ and thus satisfies the relation

$$
\left\langle\epsilon_{\lambda}^{ \pm}\right\rangle=\epsilon
$$

where $\langle\ldots\rangle$ indicates a spatial average and $\epsilon$ is the global, average dissipation rate, which is the same for $z^{+}$and $z^{-}$ fluctuations given our assumption that the cross helicity

\footnotetext{
${ }^{5}$ Equation (14) is a simplifying assumption. In numerical simulations of RMHD turbulence, $\chi^{ \pm}$has a distribution, but this distribution is scale-independent and has a mean of order unity (Mallet et al. 2014, in preparation).
} 


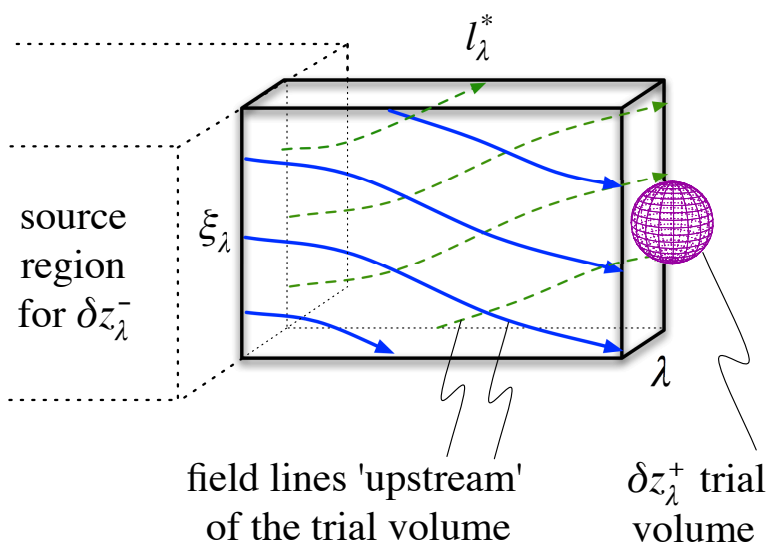

FIG. 1.- Our analysis focuses on the $z^{+}$cascade power $\left(\delta z_{\lambda}^{+}\right)^{2} / \tau_{\mathrm{nl}, \lambda}^{+}$within a spherical trial volume of diameter $\lambda$ in which $\delta z_{\lambda}^{+} \gg \delta z_{\lambda}^{*}$ and $\delta z_{\lambda}^{+} \gg \delta z_{\lambda}^{-}$. The intense $\delta z_{\lambda}^{+}$ fluctuation in the trial volume is part of a sheet-like coherent structure of thickness $\lambda$. The $\delta z_{\lambda}^{-}$fluctuation within the trial volume can be viewed as originating from a source region a distance $\sim l_{\lambda}^{*}$ from the trial volume "upstream" along the magnetic field. Field lines on opposite sides of the $\delta z_{\lambda}^{+}$structure that are initially a distance $\lambda$ apart separate by a distance $\sim \xi_{\lambda}=l_{\lambda}^{*} \delta z_{\lambda}^{+} / v_{\mathrm{A}}$ when they are followed for a distance $l_{\lambda}^{*}$.

is zero. In forced turbulence that has reached a (statistical) steady state, $\epsilon$ is also the rate at which energy is injected into the turbulence at the outer scale. Our goal now is to use Equations (18) and (19) to determine the value of $\beta$.

To average the right-hand side of Equation (18), we consider the spherical trial volume of diameter $\lambda$ illustrated in Figure 1]. We can take the PDF of $\delta z_{\lambda}^{+}$within the trial volume to be determined by Equations (15) and (71). However, once we do so, we cannot also take the value of $\delta z_{\lambda}^{-}$within the trial volume to have the same distribution, because in general $\delta z_{\lambda}^{+}$and $\delta z_{\lambda}^{-}$are correlated.

We assume that the dominant contribution to $\left\langle\left(\delta z_{\lambda}^{+}\right)^{2} \delta z_{\lambda}^{-} \sin \theta_{\lambda} / \lambda\right\rangle$ comes from exceptionally intense $\delta z_{\lambda}^{+}$fluctuations satisfying the inequalities $\delta z_{\lambda}^{+} \gg \delta z_{\lambda}^{*}$ and $\delta z_{\lambda}^{+} \gg \delta z_{\lambda}^{-}$. We therefore focus on the case in which these inequalities are satisfied within the trial volume. We assume (and confirm below in Equation (49)) that $l_{\lambda}^{+} \gg l_{\lambda}^{*}$ when $\delta z_{\lambda}^{+} \gg \delta z_{\lambda}^{*}$. Because of their comparatively large parallel correlation lengths (and long lifetimes, as we will see in Equation (47)), we refer to structures with $\delta z_{\lambda}^{+} \gg \delta z_{\lambda}^{*}$ as coherent structures.

The $z^{ \pm}$fluctuations at scale $\lambda$ propagate along a local magnetic field obtained by summing $\boldsymbol{B}_{0}$ with the magnetic-field fluctuations at length scales exceeding $\lambda$ by some factor of order unity. Because this factor is not uniquely determined, the direction in which $\delta z_{\lambda}^{ \pm}$fluctuations propagate is only determined to within an angular uncertainty of order

$$
\Delta \theta_{\lambda} \sim \frac{\delta z_{\lambda}^{*}}{v_{\mathrm{A}}}
$$

where we have taken the fluctuations at scales somewhat larger than $\lambda$ to have amplitudes comparable to $\delta z_{\lambda}^{*}$.
Here we have assumed that the intense $\delta z_{\lambda}^{+}$fluctuations propagate through a background of median-amplitude $z^{-}$fluctuations, a point that we discuss further in connection with Equation (26) below. Because of the angular uncertainty $\Delta \theta_{\lambda}$, a $z^{-}$structure of scale $\lambda$ is only able to propagate a distance $\sim \lambda /\left(\Delta \theta_{\lambda}\right) \sim l_{\lambda}^{*}$ (see Equation (17) ) through a counter-propagating $\delta z_{\lambda}^{+}$structure before propagating out of that structure.

Because of this, if we follow the magnetic-field lines in the trial volume (Figure 1) back along the magnetic field a distance $l_{\lambda}^{*}$, we reach a "source region" in which the $z^{-}$fluctuations have not yet interacted with the coherent $\delta z_{\lambda}^{+}$structure. Within this source region, the $z^{-}$ fluctuations are not aligned with the coherent $\delta z_{\lambda}^{+}$structure, because they do not yet "know about" the coherent $\delta z_{\lambda}^{+}$structure's orientation in space. If we pick two field lines a distance $\lambda$ apart within the trial volume and follow them for a distance $l_{\lambda}^{*}$, they will typically separate by a distance of order

$$
\xi_{\lambda}=\frac{l_{\lambda}^{*} \delta z_{\lambda}^{+}}{v_{\mathrm{A}}}=\lambda \frac{\delta z_{\lambda}^{+}}{\delta z_{\lambda}^{*}} \gg \lambda
$$

We assume that the coherent $\delta z_{\lambda}^{+}$structure remains coherent over a distance of at least $\sim \xi_{\lambda}$ in the direction of the vector magnetic-field fluctuations associated with the $\delta z_{\lambda}^{+}$structure - i.e., throughout the slab depicted in Figure 1, The coherent $\delta z_{\lambda}^{+}$structure is thus sheet-like.

We expect (and confirm below in Equation (47)) that the cascade timescale of the coherent $\delta z_{\lambda}^{+}$structure is $\gg$ $l_{\lambda}^{*} / v_{\mathrm{A}}$, so that the $\delta z_{\lambda}^{+}$structure changes very little as a $z^{-}$fluctuation propagates from the source region to the trial volume. We make the approximation that during this transit, the $z^{-}$fluctuation evolves as if it were acted upon by a linear $z^{+}$shear with shearing rate $\delta z_{\lambda}^{+} / \lambda$ that lasts for a time $l_{\lambda}^{*} / v_{\mathrm{A}}$, where the term "linear" refers to the shear's spatial profile (see Equation (A1)). In the Appendix, we present an analytic calculation showing that in this approximation the amplitude of the $z^{-}$fluctuation is unchanged by the shear, but the $z^{-}$fluctuation is rotated into alignment so that

$$
\sin \theta_{\lambda} \simeq \theta_{\lambda} \sim \frac{\lambda}{\xi_{\lambda}}=\frac{\delta z_{\lambda}^{*}}{\delta z_{\lambda}^{+}}
$$

within the trial volume. We also show in the Appendix that, because the $z^{-}$fluctuations are sheared at rate $\delta z_{\lambda}^{+} / \lambda$ for a time $l_{\lambda}^{*} / v_{\mathrm{A}}$, their perpendicular scales decrease by a factor of

$$
\frac{\delta z_{\lambda}^{+}}{\lambda} \times \frac{l_{\lambda}^{*}}{v_{\mathrm{A}}}=\frac{\xi_{\lambda}}{\lambda} \gg 1
$$

during their propagation from the source region to the trial volume. The source region in Figure 1 contains $z^{-}$ fluctuations spanning a range of perpendicular scales. According to the above arguments, the fluctuations at scale $\xi_{\lambda}$ in the source region make the dominant contribution to the values of $\delta z_{\lambda}^{-}$and $\delta z_{\lambda}^{-} \sin \theta_{\lambda}$ within the trial volume. Thus,

$$
\left.\left.\delta z_{\lambda}^{-}\right|_{\text {trial volume }} \simeq \delta z_{\xi_{\lambda}}^{-}\right|_{\text {source region }}
$$




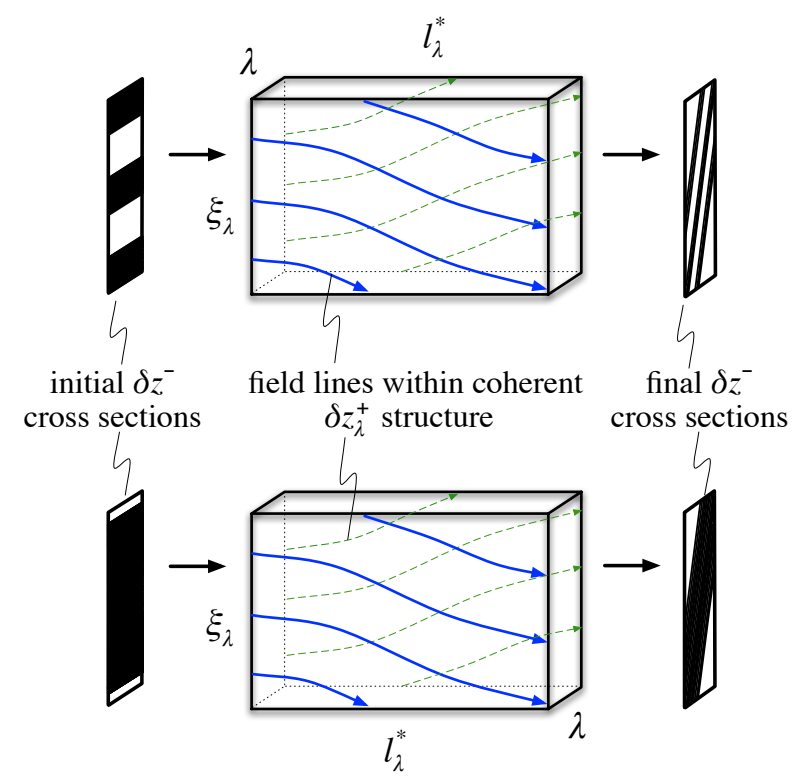

FIG. 2.- As $z^{-}$fluctuations propagate a distance $l_{\lambda}^{*}$ through the coherent $\delta z_{\lambda}^{+}$structure, the pattern of the $z^{-}$fluctuations in the field-perpendicular plane approximately follows the magnetic field lines within the coherent $\delta z_{\lambda}^{+}$structure. This causes the perpendicular length scale of the $z^{-}$fluctuations to decrease by a factor $\sim \xi_{\lambda} / \lambda \gg 1$ and rotates the $z^{-}$fluctuations into alignment with the $\delta z_{\lambda}^{+}$structure, decreasing the angle between the $\boldsymbol{z}^{+}$ and $\boldsymbol{z}^{-}$fluctuations to a value $\sim \lambda / \xi_{\lambda}$.

The top half of Figure 2 illustrates the arguments underlying Equation (22) and the scale-reduction factor in Equation (23) for the hypothetical case in which the $z^{-}$ fluctuations in the source region have square cross sections of scale $\lambda$ in the field-perpendicular plane. In the trial volume, the perpendicular scale length of these fluctuations becomes $\sim \lambda^{2} / \xi_{\lambda}$ and, because of Equation (22), $\theta_{\lambda} \ll 1$. The evolution of $z^{-}$can be recovered heuristically by taking the pattern of the $z^{-}$fluctuation in the field-perpendicular plane to follow the perturbed magnetic field lines and by taking the direction of the $\boldsymbol{z}^{-}$ fluctuation in the trial volume to become approximately parallel to the striated $z^{-}$pattern, so as to preserve the incompressibility condition. The bottom half of Figure 2 illustrates the evolution of a $z^{-}$eddy of perpendicular length scale $\sim \xi_{\lambda}$. Within the trial volume, the perpendicular length scale of this fluctuation becomes $\sim \lambda$.

Equations (22) and (24) imply that Equation (18) becomes

$$
\epsilon_{\lambda}^{+} \approx \frac{\left(\delta z_{\lambda}^{+}\right)^{2} \delta z_{\xi_{\lambda}}^{-}}{\xi_{\lambda}}
$$

when $\delta z_{\lambda}^{+} \gg \delta z_{\lambda}^{*}$, where $\epsilon_{\lambda}^{+}$and $\delta z_{\lambda}^{+}$are evaluated within the trial volume and $\delta z_{\xi_{\lambda}}^{-}$is evaluated within the source region. We now consider the average of Equation (25). Our assumption that $\delta z_{\lambda}^{+} \gg \delta z_{\lambda}^{*}$ in the trial volume decreases the probability that $\delta z_{\xi_{\lambda}}^{-}$is much larger than $\delta z_{\xi_{\lambda}}^{*}$ in the source region, because intense, counterpropagating, $z^{ \pm}$structures rapidly annihilate. We thus take the $\mathrm{PDF}$ of $\delta z_{\xi_{\lambda}}^{-}$within the source region to be neg- ligible at large $\delta z_{\xi_{\lambda}}^{-}$and make the approximation that

$$
\left.\delta z_{\bar{\xi}_{\lambda}}^{-}\right|_{\text {source region }} \simeq \delta z_{\xi_{\lambda}}^{*} .
$$

It follows from Equation (10) that $\delta z_{\xi_{\lambda}}^{*} / \xi_{\lambda}=$ $\left(\delta z_{\lambda}^{*} / \lambda\right)\left(\xi_{\lambda} / \lambda\right)^{-1-\ln \beta}$, and thus, using Equation (21), we can rewrite Equation (25) as

$$
\epsilon_{\lambda}^{+} \approx \frac{\left(\delta z_{\lambda}^{+}\right)^{1-\ln \beta}\left(\delta z_{\lambda}^{*}\right)^{2+\ln \beta}}{\lambda} .
$$

We now average Equation (27) over space. For the righthand side of Equation (27), this is equivalent to averaging over the Poisson distribution of $q$ in Equation (5), which is given in Equation (7). We thus obtain

$$
\left\langle\epsilon_{\lambda}^{+}\right\rangle \sim \frac{(\overline{\delta z})^{3}}{\lambda} \beta^{\mu(2+\ln \beta)} e^{-\mu} \sum_{q=0}^{\infty} \frac{\left(\mu \beta^{1-\ln \beta}\right)^{q}}{q !} .
$$

To derive Equation (27), we assumed that $\delta z_{\lambda}^{+} \gg \delta z_{\lambda}^{*}$, and as a consequence the form of the summand in Equation (28) is incorrect when $q \gtrsim \mu$. However, in the inertial range, in which $\mu$ is formally large, terms with $q \gtrsim \mu$ make only a small contribution to the sum in Equation (28) 6 consistent with our assumption that the total $z^{+}$dissipation rate is dominated by large- $\delta z_{\lambda}^{+}$regions. The sum in Equation (28) is simply $\exp \left(\mu \beta^{1-\ln \beta}\right)$, and thus Equation (28) implies that

$$
\left\langle\epsilon_{\lambda}^{+}\right\rangle \propto\left(\frac{\lambda}{L}\right)^{-(2+\ln \beta) \ln \beta-\beta^{1-\ln \beta}} .
$$

Since $\left\langle\epsilon_{\lambda}^{+}\right\rangle$must be independent of $\lambda$, we obtain

$$
(2+\ln \beta) \ln \beta+\beta^{1-\ln \beta}=0 .
$$

There are two solutions to Equation (30): $\beta \simeq 0.136$ and $\beta \simeq 0.691$. The solution $\beta \simeq 0.136$ leads to the scaling $\delta z_{\lambda}^{*} \propto \lambda^{1.98}$, which implies that the median variation in $\boldsymbol{z}^{ \pm}$across a distance $\lambda$ measured perpendicular to $\boldsymbol{B}$ is dominated by the outer-scale eddies and not by $\delta z_{\lambda}^{*}$, as we have assumed. Thus, the only solution to Equation (30) that is consistent with its derivation is

$$
\beta \simeq 0.691 \text {. }
$$

We note that Equations (19), (28), and (30) imply that

$$
\epsilon \sim \frac{(\overline{\delta z})^{3}}{e^{A} L} \text {. }
$$

Equation (32) establishes a relationship between the energy input into the turbulence and the two parameters $\overline{\delta z}$ and $A$ that quantify the non-universal features of the outer-scale fluctuations. Given $\epsilon$ and $L$, only one of $\overline{\delta z}$ and $A$ is a free parameter in our model.

${ }^{6}$ We verify this claim by first writing the sum in Equation (28) as $I \equiv \sum_{q=0}^{\infty}(\alpha \mu)^{q} / q !=e^{\alpha \mu}$, where $\alpha=\beta^{1-\ln \beta}<1$. For simplicity, we take $\mu$ to be an integer. The contribution to $I$ from terms with $q>\mu$ (which we denote $I_{1}$ ) then equals $e^{\alpha \mu} \gamma(\mu+1, \alpha \mu) / \mu$ !, where $\gamma$ is the lower incomplete gamma function. When $a$ exceeds $x$ by a factor of order unity and $x \gg 1, \gamma(a+1, x) \simeq e^{-x} x^{a+1} /(a-x)$ (Ferreira et al. 2005). With the use of Stirling's formula, $\mu ! \simeq$ $\sqrt{2 \pi \mu}(\mu / e)^{\mu}$, we obtain $I_{1} \simeq[\alpha /(1-\alpha)](2 \pi \mu)^{-1 / 2} \exp (\mu(1+\ln \alpha))$, which is $\ll I$ because $1+\ln \alpha<\alpha$. 


\subsection{Consistency of the Strong-Turbulence Assumption}

As described in Section 2.3, the type of nonlinear interaction that is most effective at shearing large-amplitude $\delta z_{\lambda}^{+}$structures involves the typical $\delta z_{\xi_{\lambda}}^{-} \sim \delta z_{\xi_{\lambda}}^{*}$ structures (Equation (26)) at scale $\xi_{\lambda}$, which exceeds $\lambda$ to a degree that depends on the amplitude $\delta z_{\lambda}^{+}$(see Equation (210). These are the $z^{-}$structures in the source region that, upon shearing by an intense $\delta z_{\lambda}^{+}$structure, become the $\delta z_{\lambda}^{-}$structures in the trial volume in Figure 1 (see Equation (24)) and enter into the computation of the average cascade power $\left\langle\left(\delta z_{\lambda}^{+}\right)^{2} \delta z_{\lambda}^{-} \sin \theta_{\lambda} / \lambda\right\rangle$ within the trial volume. The parallel correlation length of the typical $\delta z_{\xi}^{*}$ fluctuations in the source region in Figure 1 is $\sim l_{\xi_{\lambda}}^{*}$, and thus the correlation time of the $\delta z_{\lambda}^{-}$fluctuation in the trial volume is $\sim l_{\xi_{\lambda}}^{*} / v_{\mathrm{A}}$ (assuming that the $\delta z_{\lambda}^{+}$structure does not decorrelate on a shorter timescale, as we now demonstrate). Using Equations (22), (24), and (26), we rewrite Equation (11) in the form

$$
\tau_{\mathrm{nl}, \lambda}^{+} \sim \frac{\xi_{\lambda}}{\delta z_{\xi_{\lambda}}^{*}}=\frac{l_{\xi_{\lambda}}^{*}}{v_{\mathrm{A}}},
$$

where we have used Equation (17) to deduce that $\xi_{\lambda} / \delta z_{\xi_{\lambda}}^{*}=l_{\xi_{\lambda}}^{*} / v_{\mathrm{A}}$. Thus, the cascade timescale $\tau_{\mathrm{nl}, \lambda}^{+}$of a large-amplitude, coherent, $\delta z_{\lambda}^{+}$structure is also the correlation timescale of the $z^{-}$fluctuations that dominate the shearing of that $\delta z_{\lambda}^{+}$structure. This consistency check confirms that the turbulence is strong, as we have assumed.

\subsection{Locality}

In Equation (18), we assumed that the cascade is local in $\lambda$, in the sense that $z^{ \pm}$structures are sheared primarily by the counter-propagating $z^{\mp}$ structures of similar perpendicular scale at the same location. On the other hand, as we have just summarized in Section 2.4 an intense $\delta z_{\lambda}^{+}$fluctuation is cascaded primarily by collisions with $z^{-}$fluctuations whose perpendicular scale prior to colliding was $\xi_{\lambda}$, which significantly exceeds $\lambda$. Thus, the cascade is local in $\lambda$ if the scales of the interacting fluctuations are evaluated at the same point in space (e.g., the trial volume in Figure 1), but nonlocal if the perpendicular scale of $\delta z_{\lambda}^{+}$is evaluated in the trial volume in Figure 1 while the perpendicular scale of the $z^{-}$fluctuation is evaluated in the source region depicted in this figure. We note that Equations (15) and (33) imply that, when $\delta z_{\lambda}^{+} \gg \delta z_{\lambda}^{*}$,

$$
l_{\lambda}^{+} \sim l_{\xi_{\lambda}}^{*} .
$$

Thus, just before the nonlinear interaction begins, the $z^{-}$fluctuations that dominate the shearing of a largeamplitude, coherent $\delta z_{\lambda}^{+}$structure have the same parallel correlation length as that $\delta z_{\lambda}^{+}$structure. In this sense, the cascade could be described as "local in parallel length scale."

\subsection{Inertial-Range Scalings}

The two-point structure functions $\left\langle\left(\delta z_{\lambda}^{ \pm}\right)^{n}\right\rangle$ are the standard measures used to establish the presence of intermittency in turbulence (Kolmogorov 1962; Frisch 1996).
From Equations (5) through (9), we obtain

$$
\left\langle\left(\delta z_{\lambda}^{ \pm}\right)^{n}\right\rangle=(\overline{\delta z})^{n} e^{-\mu} \sum_{q=0}^{\infty} \frac{\left(\mu \beta^{n}\right)^{q}}{q !} .
$$

The sum in Equation (35) is simply $e^{\mu \beta^{n}}$. With the use of Equation (91), we thus obtain

$$
\left\langle\left(\delta z_{\lambda}^{ \pm}\right)^{n}\right\rangle=(\overline{\delta z})^{n}\left(\frac{\lambda}{e^{A} L}\right)^{\zeta_{n}},
$$

where

$$
\zeta_{n}=1-\beta^{n} .
$$

The summand in Equation (35) is maximized when $q \simeq$ $q_{n}$, where

$$
q_{n}=\mu \beta^{n} .
$$

Terms with $q<q_{n}$ account for approximately half of the total sum in Equation (35), just as the median of $P(q)$ in Equation (7) is approximately $\mu$ (Choi 1994). The mean value of $q$ is $\mu$, and the standard deviation of $q$ is

$$
\sigma=\left\langle(q-\mu)^{2}\right\rangle^{1 / 2}=\mu^{1 / 2} .
$$

Thus, the fluctuations that make the dominant contribution to $\left\langle\left(\delta z_{\lambda}^{ \pm}\right)^{n}\right\rangle$ are $\sim N$ standard deviations out into the tail of the $q$ distribution, where

$$
N=\frac{\mu-q_{n}}{\sigma}=\mu^{1 / 2}\left(1-\beta^{n}\right) .
$$

As $\lambda$ decreases, $N$ increases, and this increase is more rapid when $n$ is larger. It is this fact that allows $\left\langle\left(\delta z_{\lambda}^{ \pm}\right)^{n}\right\rangle$ in Equation (36) to decrease more slowly with decreasing $\lambda$ than does $\left\langle\delta z_{\lambda}^{ \pm}\right\rangle^{n}$.

From Equations (36) and (37), the second-order structure function satisfies the relation

$$
\left\langle\left(\delta z_{\lambda}^{+}\right)^{2}\right\rangle \propto \lambda^{1-\beta^{2}} \simeq \lambda^{0.52},
$$

which corresponds to an inertial-range $z^{ \pm}$power spectrum

$$
E\left(k_{\perp}\right) \propto k_{\perp}^{-1.52},
$$

where $k_{\perp}$ is the wave-vector component perpendicular to $\boldsymbol{B}_{0}$. Equation (36) implies that the kurtosis obeys the scaling

$$
\frac{\left\langle\left(\delta z_{\lambda}^{+}\right)^{4}\right\rangle}{\left\langle\left(\delta z_{\lambda}^{+}\right)^{2}\right\rangle^{2}}=\left(\frac{\lambda}{e^{A} L}\right)^{-\left(1-\beta^{2}\right)^{2}} \propto \lambda^{-0.27},
$$

which exemplifies how intermittency increases with decreasing $\lambda$. We emphasize that the parameter $A$ does not affect the exponents in any of the power-law scalings in our model (nor the fact that $\theta_{\lambda}^{\star}$ in Equation (63) below decreases logarithmically as $\lambda / L$ decreases to very small values).

For reference, Equation (10) implies that the amplitude of a "typical" structure is

$$
\delta z_{\lambda}^{*}=\overline{\delta z}\left(\frac{\lambda}{e^{A} L}\right)^{-\ln \beta} \propto \lambda^{0.37},
$$

and hence

$$
\frac{\left\langle\left(\delta z_{\lambda}^{+}\right)^{2}\right\rangle^{1 / 2}}{\delta z_{\lambda}^{*}}=\left(\frac{\lambda}{e^{A} L}\right)^{\ln \beta+\left(1-\beta^{2}\right) / 2} \propto \lambda^{-0.11} .
$$


This shows that at small $\lambda / L$ the rms fluctuation amplitude is much larger than the median fluctuation amplitude. Equation (44) implies via Equation (17) that

$$
l_{\lambda}^{*} \propto \lambda^{1+\ln \beta} \simeq \lambda^{0.63} .
$$

Equations (22), (24) and (26) imply that, when $\delta z_{\lambda}^{+} \gg$ $\delta z_{\lambda}^{*}$,

$$
\tau_{\mathrm{nl}, \lambda}^{+} \sim \frac{\lambda}{\delta z_{\lambda}^{*}}\left(\frac{\delta z_{\lambda}^{+}}{\delta z_{\lambda}^{*}}\right)^{1+\ln \beta} .
$$

The energy cascade timescale of the most intense fluctuations $\tau_{\max }$ follows from setting $\delta z_{\lambda}^{+}=\overline{\delta z}$ in Equation (47), which, together with Equation (44), yields

$$
\tau_{\max } \propto \lambda^{(1+\ln \beta)^{2}} \simeq \lambda^{0.40} .
$$

Finally, Equations (15) and (47) yield

$$
l_{\lambda}^{+} \sim l_{\lambda}^{*}\left(\frac{\delta z_{\lambda}^{+}}{\delta z_{\lambda}^{*}}\right)^{1+\ln \beta} \gg l_{\lambda}^{*}
$$

(which confirms an assumption to this effect in Section 2.3).

\subsection{Alignment}

We define the average alignment angles

$$
\theta_{\lambda}^{ \pm}=\frac{\left\langle\left|\Delta \boldsymbol{z}_{\lambda}^{+} \times \Delta \boldsymbol{z}_{\lambda}^{-}\right|\right\rangle}{\left\langle\left|\Delta \boldsymbol{z}_{\lambda}^{+}\right|\left|\Delta \boldsymbol{z}_{\lambda}^{-}\right|\right\rangle}
$$

and

$$
\theta_{\lambda}^{(v b)}=\frac{\left\langle\left|\Delta \boldsymbol{v}_{\lambda} \times \Delta \boldsymbol{b}_{\lambda}\right|\right\rangle}{\left\langle\left|\Delta \boldsymbol{v}_{\lambda}\right|\left|\Delta \boldsymbol{b}_{\lambda}\right|\right\rangle}
$$

where $\Delta \boldsymbol{v}_{\lambda}=\left(\Delta \boldsymbol{z}_{\lambda}^{+}+\Delta \boldsymbol{z}_{\lambda}^{-}\right) / 2, \Delta \boldsymbol{b}_{\lambda}=\left(\Delta \boldsymbol{z}_{\lambda}^{+}-\Delta \boldsymbol{z}_{\lambda}^{-}\right) / 2$, and $\langle\ldots\rangle$ now denotes averages over volume as well as the direction of the unit vector $\hat{\boldsymbol{s}}$ defined following Equation (3). To evaluate $\theta_{\lambda}^{ \pm}$, we set

$$
\left\langle\left|\Delta \boldsymbol{z}_{\lambda}^{+} \times \Delta \boldsymbol{z}_{\lambda}^{-}\right|\right\rangle \sim\left\langle\delta z_{\lambda}^{+} \delta z_{\lambda}^{-} \sin \theta_{\lambda}\right\rangle .
$$

We assume (and verify below) that in the inertial range the dominant contribution to $\left\langle\delta z_{\lambda}^{+} \delta z_{\lambda}^{-} \sin \theta_{\lambda}\right\rangle$ comes from regions in which $\delta z_{\lambda}^{+} \gg \delta z_{\lambda}^{*}$ or $\delta z_{\lambda}^{-} \gg \delta z_{\lambda}^{*}$. Since $\left\langle\delta z_{\lambda}^{+} \delta z_{\lambda}^{-} \sin \theta_{\lambda}\right\rangle$ is symmetric with respect to the interchange of $z^{+}$and $z^{-}$, we can estimate $\left\langle\delta z_{\lambda}^{+} \delta z_{\lambda}^{-} \sin \theta_{\lambda}\right\rangle$ by keeping only the contribution from regions in which $\delta z_{\lambda}^{+} \gg \delta z_{\lambda}^{*}$. We then evaluate this contribution by considering a spherical trial volume of diameter $\lambda$ as in Figure 1 and approximating $\delta z^{-}$and $\sin \theta_{\lambda}$ within the trial volume using Equations (22), (24), and (26). We then average over the log-Poisson PDF of $\delta z_{\lambda}^{+}$and make use of Equation (30) to obtain

$$
\left\langle\left|\Delta \boldsymbol{z}_{\lambda}^{+} \times \Delta \boldsymbol{z}_{\lambda}^{-}\right|\right\rangle \sim \overline{\delta z}^{2}\left(\frac{\lambda}{e^{A} L}\right)^{1+(\beta-1) \beta^{-\ln \beta}} \propto \lambda^{0.73},
$$

Using the same approach and setting

$$
\left\langle\left|\Delta \boldsymbol{z}_{\lambda}^{+}\right|\left|\Delta \boldsymbol{z}_{\lambda}^{-}\right|\right\rangle \sim\left\langle\delta z_{\lambda}^{+} \delta z_{\lambda}^{-}\right\rangle,
$$

we obtain

$$
\left\langle\left|\Delta \boldsymbol{z}_{\lambda}^{+} \| \Delta \boldsymbol{z}_{\lambda}^{-}\right|\right\rangle \sim \overline{\delta z}^{2}\left(\frac{\lambda}{e^{A} L}\right)^{1+\ln \beta} \propto \lambda^{0.63},
$$

Combining Equations (53) and (55), we find that

$$
\theta_{\lambda}^{ \pm} \sim\left(\frac{\lambda}{e^{A} L}\right)^{(\beta-1) \beta^{-\ln \beta}-\ln \beta} \propto \lambda^{0.10} .
$$

The above scalings reflect the contributions to $\left\langle\delta z_{\lambda}^{+} \delta z_{\lambda}^{-} \sin \theta_{\lambda}\right\rangle$ and $\left\langle\delta z_{\lambda}^{+} \delta z_{\lambda}^{-}\right\rangle$from regions in which $\delta z_{\lambda}^{+} \gg \delta z_{\lambda}^{*}$ or $\delta z_{\lambda}^{-} \gg \delta z_{\lambda}^{*}$. An upper bound on the contribution from the remaining regions in which $\delta z^{ \pm} \lesssim \delta z_{\lambda}^{*}$ can be obtained by setting $\delta z_{\lambda}^{ \pm}=\delta z_{\lambda}^{*}$ and $\sin \theta_{\lambda} \sim 1$ in $\left\langle\delta z_{\lambda}^{+} \delta z_{\lambda}^{-} \sin \theta_{\lambda}\right\rangle$ and $\left\langle\delta z_{\lambda}^{+} \delta z_{\lambda}^{-}\right\rangle$. The resulting upper bounds become negligibly small compared to the values in Equations (53) and (55) as $\lambda / L \rightarrow 0$, consistent with our assumption that the large- $\delta z^{ \pm}$regions make the dominant contributions to $\left\langle\delta z_{\lambda}^{+} \delta z_{\lambda}^{-} \sin \theta_{\lambda}\right\rangle$ and $\left\langle\delta z_{\lambda}^{+} \delta z_{\lambda}^{-}\right\rangle$.

In the inertial range, the dominant contribution to $\left\langle\left(\delta z_{\lambda}^{ \pm}\right)^{2}\right\rangle$ comes from regions in which $\delta z_{\lambda}^{ \pm}$is unusually large. In most of these regions, $\delta z_{\lambda}^{ \pm} \gg \delta z_{\lambda}^{\mp}$ and $\left|\Delta \boldsymbol{v}_{\lambda}\right| \simeq\left|\Delta \boldsymbol{b}_{\lambda}\right| \simeq \delta z_{\lambda}^{ \pm} / 2$. Keeping only the contribution to $\left\langle\left|\Delta \boldsymbol{v}_{\lambda}\right|\left|\Delta \boldsymbol{b}_{\lambda}\right|\right\rangle$ from the regions that make the dominant contributions to $\left\langle\left(\delta z_{\lambda}^{+}\right)^{2}\right\rangle$ and $\left\langle\left(\delta z_{\lambda}^{-}\right)^{2}\right\rangle$, we obtain the estimate $\left\langle\Delta v_{\lambda} \Delta b_{\lambda}\right\rangle \simeq\left\langle\left(\delta z_{\lambda}^{ \pm}\right)^{2}\right\rangle / 2$. Since $\Delta \boldsymbol{v}_{\lambda} \times \Delta \boldsymbol{b}_{\lambda}=\Delta \boldsymbol{z}_{\lambda}^{-} \times \Delta \boldsymbol{z}_{\lambda}^{+} / 2$, Equations (36) and (53) imply that

$$
\theta_{\lambda}^{(v b)} \sim\left(\frac{\lambda}{e^{A} L}\right)^{\beta^{2}+(\beta-1) \beta^{-\ln \beta}} \propto \lambda^{0.21} .
$$

Finally, we define a third average alignment angle

$$
\theta_{\lambda}^{*}=\left\langle\frac{\left|\Delta z_{\lambda}^{+} \times \Delta z_{\lambda}^{-}\right|}{\left|\Delta z_{\lambda}^{+}\right|\left|\Delta z_{\lambda}^{-}\right|}\right\rangle .
$$

The angle $\theta_{\lambda}^{*}$ is the volume average of the (sine of the) angle between $\Delta \boldsymbol{z}_{\lambda}^{+}$and $\Delta \boldsymbol{z}_{\lambda}^{-}$, whereas $\theta_{\lambda}^{ \pm}$is a weighted average of (the sine of) this angle that is dominated by regions in which the fluctuation amplitudes are large. If initially unaligned $\delta z_{\lambda}^{+}$and $\delta z_{\lambda}^{-}$fluctuations collide and $\delta z_{\lambda}^{+} \sim \delta z_{\lambda}^{-}$, then both fluctuations evolve nonlinearly on the same timescale in an unpredictable and disordered manner, which prevents the development of strong alignment. Building upon this idea, we estimate $\theta_{\lambda}^{*}$ as follows. We consider a new trial volume that is halfway between two source regions, one for $\delta z_{\lambda}^{+}$and one for $\delta z_{\lambda}^{-}$, which are separated by a distance $2 l_{\lambda}^{*}$. Because this distance is twice as large as the typical parallel correlation length that characterizes the bulk of the volume, we take $\delta z_{\lambda}^{ \pm}$in the two source regions to be statistically independent. This assumption of statistical independence breaks down for exceptionally strong fluctuations with large values of $l_{\lambda}^{ \pm}$, but such fluctuations account for only a small fraction of the volume and thus introduce only a small amount of error into our estimate of $\theta_{\lambda}^{*}$. We then set $\theta_{\lambda}=1$ in the trial volume if $\delta z_{\lambda_{1}}^{+}$and $\delta z_{\lambda_{1}}^{-}$(where $\lambda_{1}=e \lambda$ ) in the two different source regions are equal to within a factor of 3 (which is $\simeq \beta^{-3}$ ), and otherwise we set $\theta_{\lambda}=0$. We compare $\delta z_{\lambda}^{+}$and $\delta z_{\lambda}^{-}$in the two source regions at scale $\lambda_{1}$ because we assume that the fluctuations cascade from scale $\lambda_{1}$ to scale $\lambda$ as they propagate from the source regions to the trial volume. This leads 


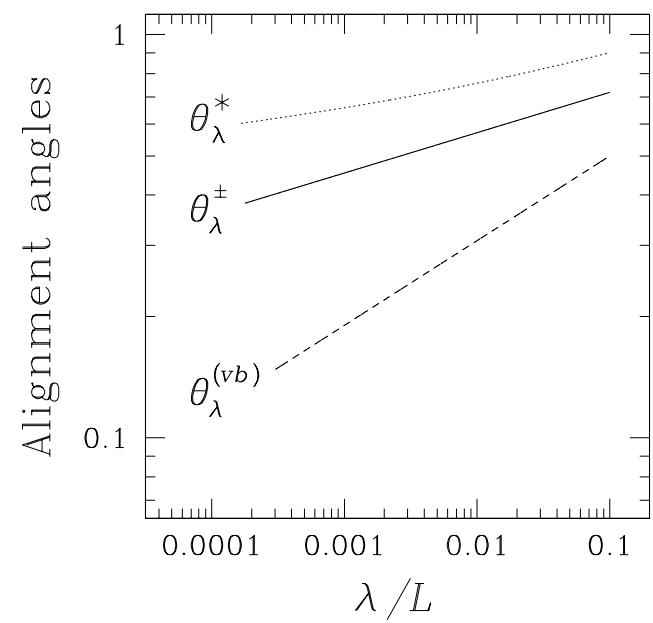

FIG. 3.- Average alignment angles $\theta_{\lambda}^{ \pm}, \theta_{\lambda}^{(v b)}$, and $\theta_{\lambda}^{*}$ defined in Equations (50), (51), and (58). The angles $\theta_{\lambda}^{ \pm}$and $\theta_{\lambda}^{(v b)}$ scale as $\lambda^{0.10}$ and $\lambda^{0.21}$, respectively. The angle $\theta_{\lambda}^{*}$ decreases as $\lambda \rightarrow 0$ more slowly than any positive power of $\lambda$. For these plots, we set $A=1$ in Equations (56) and (57), where the constant $A$ relates to the breadth of the PDF of $\delta z_{\lambda}^{ \pm}$at the outer scale. We also set $A=1$ in Equation (9) when evaluating $\mu_{1}$ in Equation (62).

to the estimate

$$
\theta_{\lambda}^{*}=e^{-2 \mu_{1}} \sum_{q_{1}=0}^{\infty} \sum_{\substack{q_{2}=0 \\\left|q_{2}-q_{1}\right| \leq 3}}^{\infty} \frac{\mu_{1}^{q_{1}+q_{2}}}{q_{1} ! q_{2} !}
$$

where

$$
\mu_{1}=\mu-1
$$

We rewrite Equation (59) in the form

$$
\theta_{\lambda}^{*}=\sum_{q_{1}=0}^{\infty} \sum_{n=0}^{3} \frac{e^{-2 \mu_{1}} \mu_{1}^{2 q_{1}+n}}{q_{1} !\left(q_{1}+n\right) !}+\sum_{q_{2}=0}^{\infty} \sum_{n=1}^{3} \frac{e^{-2 \mu_{1}} \mu_{1}^{2 q_{2}+n}}{q_{2} !\left(q_{2}+n\right) !}
$$

which is equivalent to

$$
\theta_{\lambda}^{*}=e^{-2 \mu_{1}} \sum_{n=-3}^{3} I_{n}\left(2 \mu_{1}\right),
$$

where $I_{n}(x)$ is the $n^{\text {th }}$-order modified Bessel function of the first kind. As $\lambda \rightarrow 0$,

$$
\theta_{\lambda}^{*} \propto\left[\ln \left(\frac{L}{\lambda}\right)\right]^{-1 / 2}
$$

which decreases more slowly than any positive power of $\lambda$. We plot Equations (56), (57), and (62) in Figure 3 .

\subsection{Cross Correlation}

Equations (36), (54), and (55) yield the relation

$$
\frac{\left\langle\delta z_{\lambda}^{+} \delta z_{\lambda}^{-}\right\rangle}{\left\langle\delta z_{\lambda}^{+}\right\rangle\left\langle\delta z_{\lambda}^{-}\right\rangle} \sim\left(\frac{\lambda}{e^{A} L}\right)^{\ln \beta+2 \beta-1} \propto \lambda^{0.012},
$$

which implies that $\delta z_{\lambda}^{+}$and $\delta z_{\lambda}^{-}$become anti-correlated at sufficiently small scales. However, this anti-correlation grows extremely slowly as $\lambda$ decreases. This very slow growth of anti-correlation results from the near cancellation of two competing effects. First, we argued in Equation (26) that when $\delta z_{\lambda}^{+} \gg \delta z_{\lambda}^{*}$ in the trial volume in Figure 1, the likelihood that $\delta z_{\xi_{\lambda}}^{-} \gg \delta z_{\xi_{\lambda}}^{*}$ in the source region is decreased because intense, counter-propagating fluctuations rapidly annihilate. This effect acts to make $\delta z_{\lambda}^{+}$and $\delta z_{\lambda}^{-}$anti-correlated to an increasing degree as $\lambda$ decreases, because moments of the $\delta z_{\lambda}^{ \pm}$distribution are increasingly dominated by exceptionally intense structures at smaller scales (see, e.g., the discussion following Equation (40)). On the other hand, a large-amplitude, coherent $\delta z_{\lambda}^{+}$structure amplifies $\delta z_{\lambda}^{-}$to a value $\sim \delta z_{\xi_{\lambda}}^{*}$ that exceeds $\delta z_{\lambda}^{*}$. Thus, a sheet-like coherent $\delta z_{\lambda}^{+}$structure produces a weaker, sheet-like, $\delta z_{\lambda}^{-}$-structure at the same location. On its own, this effect would act to make $\delta z_{\lambda}^{+}$and $\delta z_{\lambda}^{-}$positively correlated, to a degree that would increase at smaller scales, again because the moments of the $\delta z^{ \pm}$distribution become increasingly dominated by large-amplitude fluctuations as $\lambda$ decreases.

\section{COMPARISON WITH NUMERICAL SIMULATIONS}

The scalings in Section 2 agree reasonably well with a number of results from direct numerical simulations. For example, the $k^{-1.52}$ scaling of the inertial-range power spectrum in our model is in good agreement with the low-wavenumber ranges of the power spectra in the numerical simulations of RMHD turbulence carried out by Perez et al. (2012) and Beresnvak (2012, 2014). We note, however, that Beresnyak (2014) argued that the power spectra near the dissipation scale vary with Reynolds number in his simulations in the manner that would be expected if the inertial-range power spectrum were proportional to $k^{-5 / 3}$. A detailed discussion of this point is beyond the scope of this paper.

In Figure 4, we show the scaling exponents $\zeta_{n}$ in Equation (37) as well as the velocity-structure-function scaling exponents found by Rodriguez Imazio et al. (2013) in simulations of strong RMHD turbulence. Both sets of exponents asymptote to a value $\simeq 1$ as $n$ increases to large values. In contrast, as shown in the figure, the scaling exponents for hydrodynamic turbulence and nonhelical, globally isotropic (zero mean field), 3D, incompressible MHD turbulence reach significantly larger values at large $n$ and do not appear to asymptote towards a constant value as $n \rightarrow \infty$. We note that in the incompressible MHD simulations of Müller et al. (2003), as $\delta z_{L}^{ \pm} / B_{0}$ decreases to values $\ll 1, \zeta_{n}$ becomes increasingly similar to the RMHD results shown in Figure 4, In contrast to hydrodynamic turbulence, $\zeta_{3}$ need not be 1 in MHD turbulence or RMHD turbulence, because the average of the right-hand side of Equation (18) is not the third moment of $\delta z^{+}$, but instead a correlation function involving both $\delta z^{+}$and $\delta z^{-}$(Politano \& Pouquet 1998; Boldvrev et al. 2009).

Perez et al. (2012) found that $\theta_{\lambda}^{(v b)}$ scaled like a power law that was slightly flatter than $\lambda^{1 / 4}$ in their highestresolution, highest-Reynolds-number RMHD turbulence simulation (their simulation RB3b - see their Figure 5), consistent with Equation (57). Beresnvak (2012) found peak values of $\simeq 0.1$ for the scaling exponent $\left(d \ln \theta_{\lambda}^{ \pm}\right) / d \ln \lambda$ in numerical simulations of RMHD turbulence, consistent with Equation (56). In this same numer- 


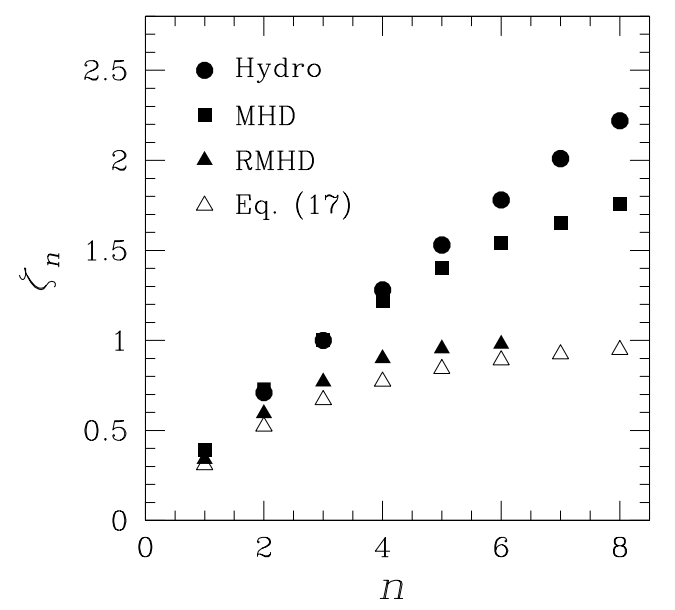

FIG. 4.- Open triangles show the predicted scaling exponents $\zeta_{n}$ of the $n^{\text {th }}$-order $z^{ \pm}$structure-function given in Equation (37). Filled triangles show the scaling exponents for the velocity structure function in numerical simulations of RMHD turbulence (Rodriguez Imazio et al. 2013). Squares show the scaling exponents of the $z^{ \pm}$structure function in numerical simulations of 3D, non-helical, zero-mean-field, incompressible MHD turbulence (Müller \& Biskamp 2000). Circles show experimentally measured scaling exponents for the velocity structure function in hydrodynamic turbulence (Benzi et al. 1993).

ical study, Beresnyak (2012) found peak values of $\simeq 0.2$ for the scaling exponent $\left(d \ln \theta_{\lambda}^{(v b)}\right) / d \ln \lambda$, in agreement with Equation (57). Beresnyak \& Lazarian (2006) carried out simulations of incompressible MHD turbulence and found that $\theta_{\lambda}^{*}$ decreased very slowly with decreasing $\lambda$, remaining close to unity throughout the inertial range in their simulations, consistent with Equation (63).

\section{DISCUSSION}

Intermittency has qualitatively different effects upon the energy cascades rates in hydrodynamic turbulence and RMHD turbulence. In hydrodynamic turbulence, an intense vorticity structure interacts with itself. The concentration of fluctuation energy into a decreasing fraction of the volume as $\lambda$ decreases thus reduces the energy cascade timescale in the energetically dominant regions, to an increasing degree as $\lambda \rightarrow 0$. Intermittency in hydrodynamic turbulence thus acts to steepen the inertial-range power spectrum. For example, $E(k) \propto k^{-1.71}$ in the SheLeveque model, whereas $E(k) \propto k^{-5 / 3}$ in Kolmogorov's (1941) theory. In RMHD, since only counter-propagating fluctuations interact, the concentration of $\delta z_{\lambda}^{+}$energy into a tiny fraction of the volume makes it difficult for a $\delta z_{\lambda}^{-}$fluctuation to "find" and interact with the dominant $\delta z_{\lambda}^{+}$fluctuations. This in turn increases the energy cascade timescale, to an increasing degree as $\lambda \rightarrow 0$, causing the inertial-range power spectrum to flatten relative to models of RMHD turbulence that neglect intermittency, a point first made by Maron \& Goldreich (2001).

Like She \& Leveque (1994) and She \& Waymire (1995), we assume that the fluctuation amplitudes have a log-Poisson PDF and make an assumption about the dimension of the most intense structures. On the other hand, the PDF of the fluctuation amplitudes (and the PDF of the dissipation rate) in the She-Leveque model has three parameters, whereas the PDF in our model has just two: $\beta$ and $\mu$ (Equations (5) and (7)). (We do not count the overall normalization of the fluctuation amplitudes - e.g., $\overline{\delta z}$ — as a parameter of the PDF in either model, because this normalization does not affect the inertial-range scalings.) Our PDF has one fewer parameter because of our argument that highly imbalanced collisions reduce a fluctuation's length scale without affecting its amplitude, which implies that the amplitude of the most intense $(q=0)$ fluctuations is independent of $\lambda$. In order to determine the extra free parameter in their model, She \& Leveque (1994) introduced an extra assumption concerning the scaling of the energy dissipation rate of the most intense structures.

In this paper, we draw heavily upon Boldyrev's $(2005,2006)$ argument that alignment within the fieldperpendicular plane plays an important role in the energy cascade. However, our treatment of scale-dependent dynamic alignment differs from Boldyrev's. In his theory, there is a single characteristic alignment angle at each scale. In our model, at each scale $\theta_{\lambda}$ varies systematically with the fluctuation amplitudes (Equation (22)). Boldyrev (2006) argued that a larger fluctuation amplitude reduces alignment. In our model, given a scale $\lambda$, larger fluctuation amplitudes are associated with enhanced alignment, a phenomenon observed by Beresnyak \& Lazarian (2006) in numerical simulations of incompressible MHD turbulence. Also, in our model, there are two distinct mechanisms for aligning $\Delta \boldsymbol{v}_{\lambda}$ and $\Delta \boldsymbol{b}_{\lambda}$ fluctuations in regions where the fluctuation amplitudes are large. First, intense $\delta z_{\lambda}^{ \pm}$fluctuations rotate weaker $\delta z_{\lambda}^{\mp}$ fluctuations into alignment, as illustrated in Figure 2, which reduces $\theta_{\lambda}^{(v b)}$ because $\Delta \boldsymbol{v}_{\lambda} \times \Delta \boldsymbol{b}_{\lambda}=\Delta \boldsymbol{z}_{\lambda}^{-} \times \Delta \boldsymbol{z}_{\lambda}^{+} / 2$. Second, when the fluctuations are intermittent, the turbulence becomes locally imbalanced at small scales (cf. Perez \& Boldvrev 2009), with either $\delta z_{\lambda}^{+} \gg \delta z_{\lambda}^{-}$or $\delta z_{\lambda}^{-} \gg \delta z_{\lambda}^{+}$in the regions containing most of the fluctuation energy. In such locally imbalanced regions, the velocity and magnetic-field fluctuations are nearly parallel or anti-parallel, regardless of whether $\boldsymbol{z}^{+}$and $\boldsymbol{z}^{-}$are aligned (Grappin et al. 2013; Wicks et al. 2013a,b). This second effect is why $\theta_{\lambda}^{(v b)}$ decreases more quickly than $\theta_{\lambda}^{ \pm}$as $\lambda / L$ decreases to small values.

Grauer et al. (1994), Politano \& Pouquet (1995), and Müller \& Biskamp (2000) developed models of intermittent, incompressible, MHD turbulence based on the approach of She \& Leveque (1994) and the assumption that $\epsilon_{\lambda}^{ \pm} \sim\left(\delta z_{\lambda}^{ \pm}\right)^{4} /\left(\lambda v_{\mathrm{A}}\right)$. Müller \& Biskamp (2000) also developed a She-Leveque-like model of incompressible MHD turbulence under the assumption that $\epsilon_{\lambda}^{ \pm} \sim\left(\delta z_{\lambda}^{ \pm}\right)^{3} / \lambda$. A major difference between our approach and these previous studies is that we set $\epsilon_{\lambda}^{ \pm} \sim$ $\left(\delta z_{\lambda}^{ \pm}\right)^{2} \delta z_{\lambda}^{\mp}\left(\sin \theta_{\lambda}\right) / \lambda$, accounting for alignment and treating $\delta z_{\lambda}^{+}$and $\delta z_{\lambda}^{-}$as separate but correlated random variables.

\section{CONCLUSION}

We have constructed an analytic model of intermittent, three-dimensional, strong RMHD turbulence that incorporates a new phenomenology of scale-dependent dynamic alignment. We restrict our analysis to the case 
of "globally balanced" turbulence, in which the cross helicity is zero. There are three main assumptions in our model. First, we take the fluctuation amplitudes to have a scale-dependent, log-Poisson PDF. In Section 2.1. we describe how this assumption can be motivated by treating a fluctuation's evolution as a random, quantized, multiplicative process, as in the work of She \& Waymire (1995). Second, we assume that the most intense $\delta z_{\lambda}^{ \pm}$ fluctuations are two-dimensional current/vorticity sheets with a volume filling factor $\propto \lambda$. Third, we assume that the turbulence obeys a refined similarity hypothesis (Equation (18) ) that includes the effect of dynamic alignment.

We argue that the largest contribution to the average $z^{+}$cascade power at any inertial-range scale $\lambda$ comes from regions in which $\delta z_{\lambda}^{+} \gg \delta z_{\lambda}^{-}$and $\delta z_{\lambda}^{+} \gg \delta z_{\lambda}^{*}$, where $\delta z_{\lambda}^{*}$ is the typical (median) fluctuation amplitude at scale $\lambda$. We then develop an approximate theory describing how a large-amplitude, coherent $\delta z_{\lambda}^{+}$structure interacts with a much weaker $z^{-}$fluctuation. We show that during such an interaction, the $z^{-}$fluctuation cascades rapidly to smaller scales without a reduction in amplitude and rotates into alignment with the coherent $\delta z_{\lambda}^{+}$structure. By accounting for these effects, we compute the average $z^{+}$cascade power using the assumed $\log$-Poisson PDF of $\delta z_{\lambda}^{ \pm}$.

This log-Poisson PDF has two free parameters, $\mu$ and $\beta$ (see Equations (5) and (9)). Our assumption that the most intense fluctuations form two-dimensional structures with a filling factor $\propto \lambda$ determines $\mu$ up to an additive constant $A$, which affects neither the power-law scalings in our model nor the fact that $\theta_{\lambda}^{*}$ (Equation (58)) decreases logarithmically as $\lambda \rightarrow 0$. The condition that the average cascade power is independent of $\lambda$ then determines $\beta$. Once we have determined $\mu$ and $\beta$, we compute the scalings of the $z^{ \pm}$power spectrum, higher-order structure functions, and three different average alignment angles. Given the assumptions stated above, the scalings in our model do not depend upon free parameters and agree reasonably well with previously published numerical results.

There are a number of ways in which our model could be improved. As presented, our model can approximate a broad distribution of outer-scale fluctuation amplitudes through the parameter $A$, but the outer-scale distribu- tion is then forced to be log-Poisson. A more realistic approach might be to allow the quantity $\overline{\delta z}$ (Equation (5)) to be random with a distribution that could be adjusted so as to model different forcing mechanisms in forced turbulence or different initial conditions in decaying turbulence. Our finding that $\tau_{\mathrm{nl}, \lambda}^{ \pm}$is an increasing function of $\delta z_{\lambda}^{ \pm}$at each scale suggests that, at least for some dissipation mechanisms such as Laplacian viscosity and resistivity, the dissipation scale is an increasing function of fluctuation amplitude. This would mean that the unusually intense fluctuations that make the dominant contribution to the power spectrum begin dissipating at a larger scale than the fluctuations that fill most of the volume. A useful direction for future research would be to develop this idea further by exploring the consequences of intermittency for the transition between the inertial and dissipation ranges within the framework of our analytic model. It would also be useful to extend our model to allow for nonzero cross helicity in order to investigate how intermittency affects strong "imbalanced" RMHD turbulence. Finally, inhomogeneity of the background plasma can fundamentally alter RMHD turbulence by causing the non-WKB reflection of Alfvén waves (Heinemann \& Olbert 1980). This linear coupling between counter-propagating Alfvén waves occurs in the solar atmosphere and solar wind (Dmitruk et al. 2002; Cranmer \& van Ballegooiien 2005; Verdini \& Velli 2007; Chandran \& Hollweg 2009) and can modify the power spectrum and energy-cascade timescales in solarwind turbulence (Velli et al. 1989; Verdini et al. 2012; Perez \& Chandran 2013). Extending our model to account for background inhomogeneity and non-WKB wave reflection would be helpful for understanding intermittent turbulence in the inner heliosphere.

This work was supported in part by grant NNX11AJ37G from NASA's Heliophysics Theory Program, NASA grant NNN06AA01C to the Solar Probe Plus FIELDS Experiment, NASA grant NNX13AF97G, and NSF grant AGS-1258998. B. Chandran was supported in part by a Visiting Research Fellowship from Merton College, University of Oxford, and a sabbatical leave from the University of New Hampshire.

\section{APPENDIX}

\section{HIGHLY IMBALANCED COLLISIONS}

In this Appendix, we consider "highly imbalanced collisions" between a large-amplitude, sheet-like, coherent, $\delta z_{\lambda}^{+}$ structure and smaller-amplitude $z^{-}$fluctuations. We begin by considering the effects of such collisions on the weaker, $z^{-}$fluctuations. For this part of our analysis, we make the simplifying approximation that the $\boldsymbol{z}^{+}$field has the form of a linear shear within the volume of the $\delta z_{\lambda}^{+}$structure. We use the term "linear" to refer to the functional form of $\boldsymbol{z}^{+}$in Equation (A1) below, and not to imply that the amplitude $\delta z_{\lambda}^{+}$is small. We further assume that the evolution of $\boldsymbol{z}^{-}$ within the volume of the coherent $\delta z_{\lambda}^{+}$structure does not depend strongly on the properties of the $\boldsymbol{z}^{+}$field outside of the structure. This assumption allows us to choose a convenient form for $\boldsymbol{z}^{+}$throughout all of space,

$$
\boldsymbol{z}^{+}=S(z, t) x \hat{\boldsymbol{y}}
$$

where $S(z, t)$ is the shearing rate and $(x, y, z)$ are Cartesian coordinates chosen so that $\boldsymbol{B}_{0}$ is in the $z$ direction. The RMHD equations can be rewritten in the form (Schekochihin \& Cowlev 2007)

$$
\frac{\partial}{\partial t} \nabla_{\perp}^{2} \psi^{ \pm} \mp v_{\mathrm{A}} \frac{\partial}{\partial z} \nabla_{\perp}^{2} \psi^{ \pm}=-\frac{1}{2}\left(\left\{\psi^{+}, \nabla_{\perp}^{2} \psi^{-}\right\}+\left\{\psi^{-}, \nabla_{\perp}^{2} \psi^{+}\right\} \mp \nabla_{\perp}^{2}\left\{\psi^{+}, \psi^{-}\right\}\right),
$$


where $\nabla_{\perp}=\hat{\boldsymbol{x}} \partial / \partial x+\hat{\boldsymbol{y}} \partial / \partial y,\{g, h\}=\hat{\boldsymbol{z}} \cdot\left(\nabla_{\perp} g \times \nabla_{\perp} h\right)$ for any functions $g$ and $h$, and $\psi^{ \pm}$are the Elsässer stream functions, which satisfy $\boldsymbol{z}^{ \pm}=\hat{\boldsymbol{z}} \times \nabla_{\perp} \psi^{ \pm}$. Equation (A1) then implies that $\psi^{+}=S x^{2} / 2$ to within an arbitrary additive function of the $z$ coordinate and time. Upon substituting this value of $\psi^{+}$into Equation (A2), we obtain

$$
\left(\frac{\partial}{\partial t}+S x \frac{\partial}{\partial y}+v_{\mathrm{A}} \frac{\partial}{\partial z}\right) \nabla_{\perp}^{2} \psi^{-}=-S \frac{\partial^{2} \psi^{-}}{\partial x \partial y} .
$$

Although $\psi^{+}=S x^{2} / 2$ is not localized, we take $\psi^{-}$to vanish sufficiently rapidly as $x^{2}+y^{2} \rightarrow \infty$ that

$$
f=\frac{1}{(2 \pi)^{2}} \int d x d y \psi^{-} e^{-i k_{x} x-i k_{y} y}
$$

is defined and the Fourier transforms in $x$ and $y$ of each term in Equation (A3) are defined. The Fourier transform of Equation (A3) yields

$$
\left(\frac{\partial}{\partial t}-S k_{y} \frac{\partial}{\partial k_{x}}+v_{\mathrm{A}} \frac{\partial}{\partial z}\right)\left(k_{\perp}^{2} f\right)=-S k_{x} k_{y} f .
$$

To solve Equation (A5), we define a family of trajectories in $k_{x}-z$ space through the equations $d k_{x} / d t=-S k_{y}$ and $d z / d t=v_{\mathrm{A}}$. The total time derivative of any function $G\left(k_{x}(t), k_{y}, z(t), t\right)$ along one of these trajectories is then $(d / d t) G=\left(\partial / \partial t-S k_{y} \partial / \partial k_{x}+v_{\mathrm{A}} \partial / \partial z\right) G$. Since $(d / d t) k_{\perp}^{2}=-2 S k_{x} k_{y}$, we can rewrite Equation (A5) as $(d / d t)\left(k_{\perp} f\right)=0$. The solution to Equation (A5) is thus

$$
f\left(k_{x}, k_{y}, z, t\right)=\frac{k_{\perp 0}}{k_{\perp}} f_{0}\left(k_{x 0}, k_{y}, z_{0}\right)
$$

where $z_{0}=z-v_{\mathrm{A}} t, k_{x 0}=k_{x}+k_{y} H$,

$$
H=\int_{0}^{t} S\left(z_{0}+v_{\mathrm{A}} t^{\prime}, t^{\prime}\right) d t^{\prime}
$$

$f_{0}\left(k_{x}, k_{y}, z\right)=f\left(k_{x}, k_{y}, z, 0\right), k_{\perp}=\sqrt{k_{x}^{2}+k_{y}^{2}}$, and $k_{\perp 0}=\sqrt{k_{x 0}^{2}+k_{y}^{2}}$. The Fourier transform of $\boldsymbol{z}^{-}$is then

$$
\boldsymbol{z}_{k}^{-}=i\left(\hat{\boldsymbol{z}} \times \hat{\boldsymbol{k}}_{\perp}\right) k_{\perp 0} f_{0}\left(k_{x 0}, k_{y}, z_{0}\right)
$$

where $\hat{\boldsymbol{k}}_{\perp}=\left(k_{x} \hat{\boldsymbol{x}}+k_{y} \hat{\boldsymbol{y}}\right) / k_{\perp}$.

If we focus on a cross section of $\psi^{-}$in the $x y$-plane, then this cross section is advected to larger $z$ at speed $v_{\mathrm{A}}$, and each Fourier component of $\psi^{-}$in that cross section is advected in $k_{x}$ at "wavenumber velocity" $-S k_{y}$, resulting in the equation

$$
k_{x}=k_{x 0}-k_{y} H
$$

where $k_{x 0}$ is the initial value of $k_{x}$. The amplitude $k_{\perp} f$ of each Fourier component of $\boldsymbol{z}^{-}$in this (moving) cross section remains constant in time. Moreover, as $t$ increases, $\left|k_{x}\right|$ may initially decrease, but eventually $\left|k_{x}\right|$ increases without bound. This causes $\hat{\boldsymbol{k}}_{\perp}$ to align with the $x$ axis and $\boldsymbol{z}^{-}$to align with the $y$ axis. The angle $\theta$ between $\boldsymbol{z}^{-}$and $\boldsymbol{z}^{+}$is $\sin ^{-1}\left|k_{y} / k_{\perp}\right|$. If $k_{x 0} \sim k_{y}$ and $H \gg 1$, then $k_{x} \simeq-k_{y} H$

$$
\theta \simeq H^{-1} \quad \text { and } \quad \frac{k_{\perp}}{k_{\perp 0}} \simeq H
$$

We now use these results to obtain an approximate description of the evolution of $z^{-}$fluctuations as they propagate a distance $l_{\lambda}^{*}$ from the source region to the trial volume depicted in Figure 1 in the limit that $\delta z_{\lambda}^{+} \gg \delta z_{\lambda}^{*}$. Because the $z^{-}$fluctuations in the source region have not yet interacted with the coherent $\delta z_{\lambda}^{+}$structure depicted in Figure 1 . they do not yet "know about" the orientation of this structure. The typical case is thus that $k_{x 0} \sim k_{y}$, so that the $z^{-}$ fluctuations are not initially aligned with the $\delta z_{\lambda}^{+}$structure. We set $S \rightarrow \delta z_{\lambda}^{+} / \lambda$, which is the shearing rate associated with the $\delta z_{\lambda}^{+}$structure, and we set $t \rightarrow l_{\lambda}^{*} / v_{\mathrm{A}}$, which is the time it takes the $z^{-}$fluctuations to propagate from the source region to the trial volume. This leads to $H=\delta z_{\lambda}^{+} / \delta z_{\lambda}^{*} \gg 1$, so that Equation (A10) applies. The condition $k_{\perp} f=$ constant and Equation (A10) then lead to Equations (22) and (24). Equation (A10) also implies that the perpendicular length scale of the $z^{-}$fluctuations decreases by a factor of $\delta z_{\lambda}^{+} / \delta z_{\lambda}^{*}$ as the $z^{-}$fluctuations propagate from the source region to the trial volume.

Finally, we consider how highly imbalanced collisions affect the larger-amplitude, coherent $\delta z_{\lambda}^{+}$structure. As can be seen in the bottom half of Figure 2, the $\delta z_{\lambda}^{-}$fluctuations within a sheet-like coherent $\delta z_{\lambda}^{+}$structure have been sheared in such a way that they resemble a smaller-amplitude, counter-propagating, current/vorticity sheet that is nearly aligned with the coherent $\delta z_{\lambda}^{+}$structure. The nature of the effect of this $\delta z_{\lambda}^{-}$current/vorticity sheet on the original $\delta z_{\lambda}^{+}$ structure is also effectively linear shearing. Because of this, we can repeat the arguments leading from Equation (A1) to Equation (A9), interchanging the roles of $z^{+}$and $z^{-}$. We thus conclude that highly imbalanced collisions between a 
sheet-like coherent $\delta z_{\lambda}^{+}$structure and much weaker $z^{-}$fluctuations change the scale but not the amplitude of the $\delta z_{\lambda}^{+}$ structure, as argued in Section 2.1. We also note that the scale of the $\delta z_{\lambda}^{+}$structure can either increase or decrease, depending on the directions of the vector fluctuations in the "colliding" fluctuations (i.e., depending on the relative signs of the two terms on the right-hand side of Equation (A9), when we have interchanged the roles of $z^{+}$and $z^{-}$in Equations (A1) through (A9) in order to describe the evolution of $\delta z_{\lambda}^{+}$).

\section{REFERENCES}

Benzi, R., Ciliberto, S., Tripiccione, R., Baudet, C., Massaioli, F., \& Succi, S. 1993, Phys. Rev. E, 48, 29

Beresnyak, A. 2012, MNRAS, 422, 3495

-. 2014, ArXiv e-prints

Beresnyak, A., \& Lazarian, A. 2006, ApJ, 640, L175

Boldyrev, S. 2005, ApJ, 626, L37

—. 2006, Physical Review Letters, 96, 115002

Boldyrev, S., Mason, J., \& Cattaneo, F. 2009, ApJ, 699, L39

Boldyrev, S., Nordlund, Å., \& Padoan, P. 2002a, ApJ, 573, 678

—. 2002b, Physical Review Letters, 89, 031102

Bruno, R., Carbone, V., Chapman, S., Hnat, B., Noullez, A., \& Sorriso-Valvo, L. 2007, Physics of Plasmas, 14, 032901

Burlaga, L. F. 1991, J. Geophys. Res., 96, 5847

Chandran, B. D. G., \& Hollweg, J. V. 2009, ApJ, 707, 1659

Cho, J., \& Vishniac, E. T. 2000, ApJ, 539, 273

Choi, K. P. 1994, Proc. Amer. Math. Soc., 62, 242

Cranmer, S. R., \& van Ballegooijen, A. A. 2005, ApJS, 156, 265

Dmitruk, P., Matthaeus, W. H., Milano, L. J., Oughton, S., Zank, G. P., \& Mullan, D. J. 2002, ApJ, 575, 571

Dubrulle, B. 1994, Physical Review Letters, 73, 959

Ferreira, C., López, J. L., \& Sinusía, E. P. 2005, Advances in Applied Mathematics, 32, 467

Forman, M. A., \& Burlaga, L. F. 2003, in American Institute of Physics Conference Series, Vol. 679, Solar Wind Ten, ed. M. Velli, R. Bruno, F. Malara, \& B. Bucci, 554-557

Frisch, U. 1996, Turbulence

Frisch, U., Sulem, P.-L., \& Nelkin, M. 1978, Journal of Fluid Mechanics, 87, 719

Galtier, S., Nazarenko, S. V., Newell, A. C., \& Pouquet, A. 2000, Journal of Plasma Physics, 63, 447

Goldreich, P., \& Sridhar, S. 1995, ApJ, 438, 763

Grappin, R., Müller, W.-C., Verdini, A., \& Gürcan, Ö. 2013, ArXiv e-prints

Grauer, R., Krug, J., \& Marliani, C. 1994, Physics Letters A, 195 , 335

Greco, A., Valentini, F., Servidio, S., \& Matthaeus, W. H. 2012, Phys. Rev. E, 86, 066405

Gurvich, A. S., \& Yaglom, A. M. 1967, Physics of Fluids, 10, 59

Heinemann, M., \& Olbert, S. 1980, J. Geophys. Res., 85, 1311

Horbury, T. S., \& Balogh, A. 1997, Nonlinear Processes in Geophysics, 4, 185

Iroshnikov, P. S. 1963, AZh, 40, 742

Kadomtsev, B. B., \& Pogutse, O. P. 1974, Soviet Journal of Experimental and Theoretical Physics, 38, 283

Karimabadi, H., et al. 2013, Physics of Plasmas, 20, 012303

Kolmogorov, A. N. 1941, Dokl. Akad. Nauk SSSR, 30, 299

Kolmogorov, A. N. 1962, Journal of Fluid Mechanics, 13, 82

Kraichnan, R. H. 1965, Physics of Fluids, 8, 1385

Maron, J., \& Goldreich, P. 2001, ApJ, 554, 1175

Mininni, P. D., \& Pouquet, A. 2009, Phys. Rev. E, 80, 025401

Müller, W.-C., \& Biskamp, D. 2000, Physical Review Letters, 84, 475

Müller, W.-C., Biskamp, D., \& Grappin, R. 2003, Phys. Rev. E, 67,066302
Ng, C. S., \& Bhattacharjee, A. 1996, ApJ, 465, 845

Osman, K. T., Kiyani, K. H., Chapman, S. C., \& Hnat, B. 2014 ApJ, 783, L27

Osman, K. T., Matthaeus, W. H., Hnat, B., \& Chapman, S. C.

2012, Physical Review Letters, 108, 261103

Paladin, G., \& Vulpiani, A. 1987, Phys. Rep., 156, 147

Parisi, G., \& Frisch, U. 1985, Turbulence and Predictability,

Varenna Summer School, ed. M. Ghol, R. Benzi, \& G. Parisi (Amsterdam: North-Holland)

Perez, J. C., \& Boldyrev, S. 2009, Physical Review Letters, 102, 025003

Perez, J. C., \& Chandran, B. D. G. 2013, ApJ, 776, 124

Perez, J. C., Mason, J., Boldyrev, S., \& Cattaneo, F. 2012, Physical Review X, 2, 041005

Perri, S., Carbone, V., Vecchio, A., Bruno, R., Korth, H., Zurbuchen, T. H., \& Sorriso-Valvo, L. 2012, Physical Review Letters, 109, 245004

Politano, H., \& Pouquet, A. 1995, Phys. Rev. E, 52, 636

—. 1998, Phys. Rev. E, 57, 21

Rodriguez Imazio, P., Martin, L. N., Dmitruk, P., \& Mininni, P. D. 2013, Physics of Plasmas, 20, 052506

Schekochihin, A. A., \& Cowley, S. C. 2007, Turbulence and Magnetic Fields in Astrophysical Plasmas, ed. S. Molokov, R. Moreau, \& H. K. Moffatt (Springer), 85

Schekochihin, A. A., Cowley, S. C., Dorland, W., Hammett, G. W., Howes, G. G., Quataert, E., \& Tatsuno, T. 2009, ApJS, 182,310

Servidio, S., Valentini, F., Califano, F., \& Veltri, P. 2012,

Physical Review Letters, 108, 045001

She, Z.-S., \& Leveque, E. 1994, Physical Review Letters, 72, 336

She, Z.-S., \& Waymire, E. C. 1995, Physical Review Letters, 74, 262

Shebalin, J. V., Matthaeus, W., \& Montgomery, D. 1983, Journal of Plasma Physics, 29, 525

Sorriso-Valvo, L., Carbone, V., Veltri, P., Consolini, G., \& Bruno, R. 1999, Geophys. Res. Lett., 26, 1801

Strauss, H. R. 1976, Physics of Fluids, 19, 134

Velli, M., Grappin, R., \& Mangeney, A. 1989, Physical Review Letters, 63, 1807

Verdini, A., Grappin, R., Pinto, R., \& Velli, M. 2012, ApJ, 750, L33

Verdini, A., \& Velli, M. 2007, ApJ, 662, 669

Wan, M., Osman, K. T., Matthaeus, W. H., \& Oughton, S. 2012a, ApJ, 744, 171

Wan, M., et al. 2012b, Physical Review Letters, 109, 195001

Wicks, R. T., Mallet, A., Horbury, T. S., Chen, C. H. K., Schekochihin, A. A., \& Mitchell, J. J. 2013a, Physical Review Letters, 110, 025003

Wicks, R. T., Roberts, D. A., Mallet, A., Schekochihin, A. A., Horbury, T. S., \& Chen, C. H. K. 2013b, ApJ, 778, 177 Wu, P., et al. 2013, ApJ, 763, L30 